\title{
A COMPOSITIONAL SEMANTICS OF TEMPORAL EXPRESSIONS IN ENGLISH
}

\author{
Erhard W. Hinrichs \\ BBN Laboratories Inc. \\ 10 Moulton St. \\ Cambridge, MA 02238
}

\begin{abstract}
This paper describes a compositional semantics for temporal expressions as part of the meaning representation language (MRL) of the JANUS system, a natural language understanding and generation system under joint development by BBN Laboratoires and the Information Sciences Institute. ${ }^{1}$ The analysis is based on a higher order intensional logic described in detail in Hinrichs, Ayuso and Scha (1987). Temporal expressions of English are translated into this language as quantifiers over times which bind temporal indices on predicates. The semantic evaluation of time-dependent predicates is defined relative to a set of discourse contexts, which, following Reichenbach (1947), include the parameters of speech time and reference time. The resulting context-dependent and multi-indexed interpretation of temporal expressions solves a set of well-known problems that arise when traditional systems of tense logic are applied to natural language semantics. Based on the principle of rule-to-rule translation, the compositional nature of the analysis provides a straightforward and welldefined interface between the parsing component and the semantic interpretation component of JANUS.
\end{abstract}

\section{Introduction}

JANUS is a natural language understanding and generation system which allows the user to interface with several knowledge bases maintained by the US NAVY. The knowledge bases contain, among other things, information about the deployment schedules, locations and readiness conditions of the ships in the Pacific Fleet.

(1) a. Did the admiral deploy the ship?

b. Which $\mathrm{C} 3$ ships are now $\mathrm{C4}$ ?

c. When will Vincent arrive in Hawaii?

d. Who was Frederick's previous commander?

As the sample queries in (1) demonstrate, much of

\footnotetext{
'The work presented here was supported under DAAPA contract \#N00014-85-C-0016. The views and conclusions contained in this document are those of the authors and should not be interpreted as necessarily representing the official policies, either expressed or implied, of the Defense Advanced Research Projects Agency of of the United States Govemment.
}

this information is highly time-dependent: Ships change locations in accordance with their deployment schedules, incur equipment failures or undergo personnel changes which can lead to changes in the ship's readiness rating. It is, therefore, imperative that at the level of semantic representation of the natural language input an adequate analysis can be provided for those linguistic expressions that carry time information, for example, tenses, temporal adverbials and temporal adjectives.

\section{Applying Classical Tense Logic To Natural Language Semantics}

My own treatment of temporal expressions is very much a response to the kinds of analyses that have been provided in classical tense logic. When I refer to classical tense logic I mean the kinds of logics that orginate in the work of the logician Arthur Prior (Prior 1967) and that have been applied by Montague (Montague 1973) and others to natural language semantics.

In classical tense logic time-dependency of information enters into the definition of the notion of a proposition. Propositions are defined as functions from a set of times $\mathrm{TI}$ to the set of truth values true and false. Declarative sentences of natural language are taken to express propositions. The sentence it is raining can be taken to be that proposition which yields the value true for those times at which it is raining and false for those at which it is not.

Tense operators can be defined in such a logic as in (2) and (3). (2) defines a past operator capital $P$ which, applied to a proposition $p$, yields the value true for some time $t$ if the proposition $p$ is true at some time t' prior to t. Likewise. (3) defines a $Y$ operator. where $Y$ is mnemonic for yesterday. with the expected truth conditions: $Y p$ is true at $t$ if $p$ is true at some time $t$ ' that falls within the prior to the day in which $t$ falls.

(2) $[P p]_{t}=T$ iff $[p]_{t}=T$ for some time $t^{\prime}<t$.

(3) $[Y p]_{p}=T$ iff $[p]_{p}=T$ for some time $t^{\prime} \varepsilon[D A Y(t)-1]$.

All of this sounds rather plausible. However, it turns out that if one tries to apply tense operators such as $P$ and $Y$ in natural language semantics, a set of well- 
known problems arise. ${ }^{2}$

\section{Inadequacies Of Classical Tense Logic}

\subsection{Interaction of Tense and Time Adverbials}

The first such problem, which I pointed out in $\mathrm{Hin}$ richs (1981) and which has been independently noted by Dowty (1982), concerns the interaction between tense and time adverbials. If for sentence (4), one interprets the past tense in (4) by the $P$ operator and the adverbial yesterday by the $Y$ operator, then one of the two operators has to have scope over the other.

(4) Vincent left yesterday.

(5) $P\left[Y\left[\right.\right.$ leave $\left(\right.$ Vincent') $\left.\left.^{\prime}\right]\right]$

(6) $Y[P[$ leave' (Vincent') $]]$

However, neither the formula in (5), nor the one in (6) gives adequate truth conditions for (4). In (5) the $P$ operator shifts the temporal evaluation of the proposition Ylleave'(Vincent')] from the speech time to some past time $t^{\prime}$ and then the $Y$ operator shifts evaluation to some time $t^{n}$ within the day prior to $t^{\prime}$, instead of the day prior to the speech time. (6) assigns wrong truth conditions as well. Here the $Y$ operator shitts evaluation to some time within the day prior to the speech time. But then the $P$ operator in turn shift evaluation to some time prior to that, but necessarily within the same day.

\subsection{Interaction of Tense and Negation}

Similar problems arise when one uses standard tense logic for sentences in which tense interacts with sentence negation as in (7). As was first pointed out by Parte日 (1973), one can assign the past tense operator $P$ either narrow scope with respect to nega. tion as in (8) or wide scope as in (9).

(7) Vincent did not leave.

(8) $\neg[P[$ leave' (Vincent') ]]

(9) $P[\neg[$ leave'(Vincent') ]]

However, neither the formula in (8), nor the one in (9) assigns adequate truth conditions to (7). Formula (8) says that there exists no time in the past at which the proposition is true, clearly not capturing the meaning of (7). (9) makes (7) true if at any time in the past

\footnotetext{
In fairness to Prior, it has to be pointed out thet he designed his temporal modal logics as purely formal systems and did not design them with idea of applying them to natural language. However. Priorean tense logic has, nonetheless, been applied to natural language semantics. It is those studies that are subject to the criticisms presented in sections 3.1 - 3.4
}

Vincent did not leave. Given that ships participate in events other than arrivals at some point during their existence, (9) will be trivially satified, but does not capture adequately the truth conditions of (7).

\subsection{Tense and Quantified NP}

The third type of inadequacy of standard tense logic has to do with the interaction of tense and quantified NP's and was first pointed out by Enc (1981). Enc points out that Priorean tense operators fail to capture certain readings of sentences such as (10).

(10) Every admiral was (once) a cadet.

(11) $\forall x\left[\operatorname{admiral}(x) \rightarrow P\left[\operatorname{cadet}^{\prime}(x)\right]\right]$

(12) $P\left[\forall x\left[\operatorname{admiral}(x) \rightarrow \operatorname{cadet}^{\prime}(x)\right]\right]$

Since the past tense operator $P$ is a propositional operator. it can take scope over the consequent of the material implication in (11). (11) represents the reading that everyone who is an admiral now was a cadet at some time in the past. The second reading in (12), where $P$ has scope over the entire formula assigns the somewhat absurd truth conditions that at some time in the past every admiral as simultaneously a cadet. However, as Enc observes correctly, with propositional tense operators one cannot obtain the perfectly natural reading that everyone who is an admiral now or who was an admiral at some time in the past was a cadet at some time prior to being an admiral.

\subsection{Temporal Anaphora}

There is fourth problem that arises when one uses tense operators of standard tense logic for the semantic interpretation of single sentences or pieces of discourse that describe multiple events.

(13) Vincent was hit by a harpoon, was abandoned by its crew, and sank.

The most natural interpretation of (13) is one in which the events are understood to have happened in the same temporal order as they are sequenced in the sentence. However, if one uses a Priorean $P$ operator to interpret each occurrence of the past tense in (13), one arrives at an interpretation. which incorrectly allows for any temporal ordering.

\section{A Tense Logic with Multiple Indices}

It turns out that most of the problems that I have just discussed can be solved if one recognizes more than one parameter of temporal evaluation. In the models given to tense logics such as the ones first 
developed by Prior, one standardly evaluates propositions with respect to a single time which one may call the event time, the time at which an event happens of at which a state of affairs obtains. The point of sperch is taken to be a special case of this parameter.

An alternative to models with only one temporal parameter has been given by Reichenbach (1947). Reichenbach argues for distinguishing between three parameters which he calls speech time, event time and reference time. The meaning of the first two parameters should be self-explanatory. It is the third parameter, reference time, that requires explanation. Reichenbach conceives of reference time as the temporal perspective from which an event is viewed, as opposed to event time as the time at which the event occurs. Reference time can be either implicit in the discourse context or explicitly specified by temporal adverbials such as yesterday. For each individual tense reference time is temporally ordered with respect to the other two parameters. Reference time plays a crucial role in Reichenbach's account of the distinction betwen the simple past and the present perfect in English. In both cases event time prece日ds speech time. But while for the simple past, the event time is viewed from a perspective in the past, the event is viewed from the perspective of the present in the case of the present perfect.

Given the distinction between reference time and event time. one can then formalize Reichenbach's analysis of the past tense as in (14). The operator $P$ shifts evaluation of the event time $t$ to some time $t$ ' in the past such that $t^{\prime}$ falls within some reference time $r$.

(14) $[P p]_{r, t}=T$ iff $[P]_{P, r}$ for some time $t$ ' such that $t^{\prime}<t$ and $t^{\prime} \subseteq r$.

The $Y$ operator on the other hand, does not shift the event time $t$, rather it operates on the reference time $r$ in the obvious way.

$$
\text { (15) }[Y p]_{r, t}=T \text { iff }[P]_{[D A Y(t)-1], t}=T \text {. }
$$

With the redefined operators $P$ and $Y$, one can now give adequate truth conditions for sentences involving tense and time adverbials. In the formula in (16) $Y$ specifies the reference time $r$ to be the day prior to the speech time, and then the $P$ operator locates the event time as being within that reference time.

$$
\begin{aligned}
& \text { (16) }\left[Y \left[P[\text { leave' }(\text { Vincent') }]]_{r, t}=T\right.\right. \\
& \text { iff }\left[P[\text { leave' }(\text { Vincent') }]]_{\left[D A Y\left(t_{s}\right)-1\right], t}=T\right. \\
& \text { iff [ leave' }(\text { Vincent') }]_{\left[D A Y\left(t_{s}\right)-1\right], t^{\prime}}=T \text { for } \\
& \text { some } t^{\prime}<t \text { and } t^{\prime} \subseteq\left[D A Y\left(t_{s}\right)-1\right] .
\end{aligned}
$$

Likewise for tense and negation, the past operator locates the event time $t$ prior to speech time and within some reference time $r$ which in the case of (17) has to be taken to be contextually specified.

\footnotetext{
${ }^{3}$ Operators similar to the redefined $P$ and $Y$ operators have first been suggested in the literature by Acquist (1976).
}

(17) Vincent did not leave.

(18) $\left[\neg\left[P[\text { leave'(Vincent')] }]_{r, t}=T\right.\right.$ iff [ P[leave'(Vincent')] $]_{r, t}=F$ iff [leave'(Vincent') $]_{r, t}=F$ for all times $t^{\prime}$ such that $t^{\prime}<t$ and $t^{\prime} \subseteq r$.

(17) is true according to (18) if there is no time within the reference time $r$ at which the untensed proposition leave'(Vincent') is true.

It turns out that a multi-indexed tense logic also gives an adequate account of tense in discourse. A detailed account of this can be found in Hinrichs (1981, 1986); here I will only sketch the basic idea: By ordering event times with respect to reference times, as sketched in (20), and by updating such reference times after each event description, one can order multiple events as described in (19) in the appropriate way. The relations $<$ and $\subseteq$ in $(20)$ are meant to stand for temporal precedence and temporal inclusion, respectively.

(19) Vincent [was hit by a harpoon $]_{\theta_{1}}$, [was abandoned by its crew $]_{\theta_{2}}$, and $[\operatorname{sank}]_{\theta_{3}}$.

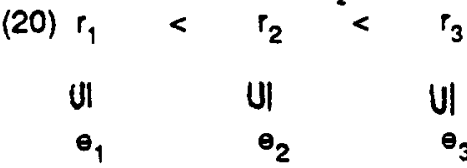

Let us consider next two alternative logical representations for sentence (21) in such a multiindexed logic.

(21) Vincent left yesterday.

(22) $\left[Y\left[P[\text { leave' (Vincent') ] ] }]_{r, t}\right.\right.$

(23) $\exists t^{\prime}\left[t^{\prime}<t_{s} \& t_{r}=\left[D A Y\left(t_{s}\right)-1\right] \& t^{\prime} \varepsilon t_{r}\right.$ \& leave $\left(\right.$ Vincent' $\left.\left.^{\prime}\right)\left(t^{\prime}\right)\right]$

The one in (22) I have already discussed. In (22) past tense is translated into a propositional operator whose semantics is implicit in the truth conditions imposed with respect to the model-theory. In the formula in (23) the past tense leads to existential quantification over times. The existential quantifier binds variables which appear as extra argument positions on predicates. So, ship which is ordinarily taken to be a one-place predicates turns into a two-place predicate that takes individuals and times as its arguments. The variable $t$, occurs as a free variable in (23) and stands for the Reichenbachean reference time.

Although the two formulas in (22) and (23) are logically equivalent in the sense that both are true under the same set of models, I will adopt the style of logical representation in (23) for remainder of this paper This is because in the context of the JANUS system, it is important to explicitly quantify over times since in the database times are explicitly entered as dates, time stamps, etc. In order to be able to access them, it is important to incorporate time information explicitly at the level of logical form.

A second reason for preferring the style of 
representation in (23) over the one in (22) concerns the interaction between tenses and quantified NP's. Since formulas such as (23) explicitly quantify over times, scope relations with respect to quantification over individuals become completely transparent.

\section{Tense and Quantified Noun Phrases}

Using the style of representation exemplified by formula (23), let me then return to the issue of tense and quantification, which is still unresolved. Consider once again the types of examples that, as Enc points out, cannot be handled in standard tense logic.

(24) Every admiral was (once) a cadet.

(25) $\forall x\left[\operatorname{admiral}(x) \rightarrow P\left[\operatorname{cadet}^{\prime}(x)\right]\right]$

(26) $P\left[\forall x\left[\operatorname{admiral}(x) \rightarrow \operatorname{cadet}^{\prime}(x)\right]\right]$

If tense operators like $P$ have scope over propositions, $P$ can either scope over an entire formula as in (25) or over the consequent of the material implication as in (26). Now, as we saw earlier, neither formula captures the reading that all present or past admirals were cadets prior to their being admirals.

Enc (1981) provides an interesting solution to the problem posed by examples such as (24). Her solution is based on two assumptions: 1 . Semantically, tenses should have scope only over verb meanings, but not over any larger elements in a sentence, and 2. verb meanings as well as noun meanings are indexical in the sense their interpretations depend on the context of the utterance in the same way that demonstrative pronouns such as that and anaphoric pronouns such as she and they do.

As the formula in (27) shows, which represents the translation for (24) in my analysis, I adopt Enc's first assumption and assign tense scope only over the main verb of the sentence.

(27) $\forall x[\exists t[$ admiral' $(x)(t) \& R(x)(t)] \rightarrow$ $\left[\exists t^{\prime}\left[t^{\prime}<t_{s} \& t^{\prime} \varepsilon t_{,} \&\right.\right.$ graduate-from'(WestPoint $\left.\left.)(x)\left(t^{\prime}\right)\right]\right]$

The predicate $R$ in (27), whose role I will comment on in more detail shortly, is meant to range over properties which are salient in a given context. The past tense of sentence (24) contributes the existential quantification over times that precede the speech point $t_{s}$ and are contained in some contextually specified reference time $t_{r}$. Following Enc, tense is thus given scope only over the predicate that corresponds to the main verb. However, the formula in (27) also shows that I do not follow Enc in her second assumption, namely her treatment of nouns as indexicais. In contrast to true indexicals, whose denotation depends solely on the context of utterance, I treat the denotation of predicates corresponding to nouns as being time-dependent in an absolute sense, since predicates such as admiral do carry a time-denoting argument position as part of their function-argument structure. Without such an argument, it seems impossible to give a satisfactory account of temporal adjectives such as former and previous or last, whose function it is to shift the temporal evaluation of the predicate that they combine with. However, I do recognize an element of context dependency inherent in the interpretation of noun phrases such as every admiral, since I interpret such noun phrases with respect to some contextually salient property $R$. This predicate makes it possible to account for the well-known phenomenon of restricted quantification, namely that in sentences such as (28) the interpretation of everyone does not involve the set of all students in the world, but rather the set of all individuals in a given context; for example everyone at a certain party. ${ }^{4}$

(28) Everyone is having a good time.

Temporal evaluation of the verbal predicate is, thus, kept separate from the temporal evaluation of predicates corresponding to other constituents in the sentence. As first pointed out by Enc, this strategy makes it possible to account for sentences such as (29) and (30) whose translations require that the predicates secretary and fugitive be evaluated relative to a time which is distinct from the evaluation time of the predicate corresponding to the verb. ${ }^{5}$

(29) Oliver North's secretary testified before the committes.

(30) Every fugitive is now in jail.

In contrast to an analysis which interprets the past tense in terms of a Priorean $P$ operator, the narrow scope analysis of tense also avoids the dilemma of inducing a simultaneity reading for sentence (31), if the tense operator $P$ has scope over the entire formula as in the translation (32) of (31).

(31) Every admiral graduated from West Point.

(32) $P$ [ $\forall x$ [admiral' $(x) \rightarrow$ graduate-from' (WestPoint' $)(x)]]$

The reading in (32) is factually implausible for two reasons: 1. It imposes simultaneity as part of the truth conditions and requires that all admirals graduated at the same time, 2. since the P operator forces temporal evaluation of all predicates in its scope at the same index, in the case of (31) it requires that every admiral graduated from West Point as an admiral, and not, as is actually the case, subsequent to graduation from the Naval academy.

Notice that the formula in (33), which represents the translation of (31) in my analysis, avoids both problems associated with (32).

(33) $\forall x[\exists t[$ admiral'(x)(t) \& $R(x)(t)] \rightarrow$ $\left[\exists t^{\prime}\left[t^{\prime}<t_{s} \& t^{\prime} \& t_{r} \&\right.\right.$ graduate-from'(WestPoint $\left.\left.)(x)\left(t^{\prime}\right)\right]\right]$

\footnotetext{
The example is due to Stainaker (1973).

SRecall that Fawn Hall, North's secretary, testified before the committee when she was no longer North's secretary. The example is due to an editorial in the Boston Giobe
} 
Since temporal evaluation of the predicates admiral' and graduate-from are kept separate, the first problem does not arise. Since the predicates are existentiaily quantified over independently, (33), in contrast to (32), also avoids having to assign a simultaneity reading to (31).

A crucial element of my analysis is the inclusion of the predicate $R$, which is meant to restrict the denotation of quantified NP 's such as every ship by properties that are salient in the context of utterance. Apart from keeping the temporal evaluation of verbal predicates and nominal predicates independent of one another, it is this context dependent feature of my analysis that makes it more flexible than a wide scope analysis of tense. Let me illustrate how the contextdependent evaluation of quantified NP's by once again focusing on example (34).

(34) Every admiral graduated from West Point.

Imagine that (34) is uttered in a context in which all current admirals assigned to the Pacific Fleet are under discussion. In that context, $R$ could be instantiated as in (35), i.e. as the intension of the set of individuals $x$ which are assigned to the Pacific Fleet at a time which equals the speech time $t_{s}$.

(35) $\lambda t \lambda y$ [assigned-to'(Pac-Fle日t') $(y)(t) \& t=t_{s}$ ]

Substituting $R$ by (35) in (36), one then arrives at the formula in (37).

(36) $\forall x[\exists t[$ admiral'(x)(t) \& $R(x)(t)] \rightarrow$ $\left[\exists t^{\prime}\left[t^{\prime}<t_{s} \& t^{\prime} \varepsilon t_{r} \&\right.\right.$ graduate-from'(WestPoint $\left.)(x)\left(t^{\prime}\right)\right]$

(37) $\forall x[\exists t[$ admiral' $(x)(t) \&$ assigned-to'(PacFleet' $\left.)(x)(t) \& t=t_{s}\right] \rightarrow\left[\exists t^{\prime}\left[t^{\prime}<t_{s} \& t^{\prime} \varepsilon t_{p} \&\right.\right.$ graduate-from'(West-Point')( $x)\left(t^{\prime}\right)$ ]]

In a context in which all present or past admirals in the Pacific Fleet are under discussion, a reading which, as 1 pointed out in section 3.3, one cannot capture using Priorean tense operators one can capture by instantiating $R$ as in (38), where $\leq$ stands for the relation temporally preceding or equal to.

\section{(38) $\lambda t \lambda y$ [assigned-to'(Pac-Fleet' $)(y)(t) \& t \leq t_{s}$ ]}

The idea behind using the variable $R$ in my analysis is, thus, to have it instantiated appropriately by the discourse context. One of the counterarguments that one may raise against this context-dependent aspect of my analysis of temporal semantics concerns the fact that tracking the salience of objects and their properties in natural language discourse is a notoriously difficult problem. However, I will argue in the next section that whatever mechanisms are needed to track saliency, such mechanisms are motivated independently by semantic and pragmatic phenomena that go beyond phenomenon of temporal interpretation.

\section{Evaluating Time-dependent Predicates in Context}

Objects and certain of their properties can receive or maintain salience in a discourse in any number of ways. The notions of focus (Sidner 1983), of common ground (Stalnaker 1978) and of mutual knowledge (Clark and Marshall 1981) are certainly cases in point. In this section I will concentrate on one such mechanism which plays a role in the contextdependent interpretation of time dependent predicates. I will argue that the mechanism is needed for purposes other than temporal interpretation and, therefore, does not add complexity to my analysis of temporal semantics.

Consider a typical sequence of queries that a user may present to JANUS.

(39) a. Did every admiral deploy a ship yesterday?

b. Which ships will arrive in Hawaii?

The person asking (39b) is not interested in being informed about all ships that at some time in the future will go to Hawaii. Instead, the user is interested in a much more restricted set of ships that will go there, namely the ones that were deployed by some admiral the day before. In order to arrive at such an interpretation, the free variable $R$ in the translation formula in (40) has to be bound appropriately by the context.

(40) QUERY [ $\lambda$ z [ $z$ \& POW[ $\lambda$ y $\exists t^{\prime}\left[\operatorname{ship}^{\prime}(y)\left(t^{\prime}\right)\right.$

$\left.\left.\& R(y)\left(t^{\prime}\right)\right]\right] \& \exists t\left[t>t_{s} \& t \in t_{r}\right.$

\& go-to'(Hawaii')(z)(t) $]_{t_{i}}$ ]

QUERY is a speech act operator which takes the propositional content of the question as an argument and causes to evaluate it at some temporal index, in this case the point of speech $t_{s}$. In (40) QUERY applies to a lambda-abstract over those sets of objects $x$ which are the speech time $t_{s}$ in the Indian Ocean and whose members y at some time thave the property of being a ship and which are in addition distinguished by some contextually salient property $R$. POW stands for the power set operation which I use for the interpetation of plural nouns. Now if the reader prefers some other approach to the semantics of plurals, say the lattice-theoretic approach of Link (1983), over the approach based on power sets I am not going to argue with them. The point that I want to concentrate on with respect to the formula in (40) concerns the instantiation of the context-dependent predicate R. The predicate ship' has to be interpreted relative to the discourse context. and the temporal evaluation of the predicate is determined with respect to that context, rather by the tense of the sentence, in this case the future.

It turns out that a detailed proposal for how to track objects and their properties does, in fact, already exist in the literature. In her work on the interpretation 
of pronouns in discourse, Webber (1978.1983) has developed a framework that constructs during the interpretation of a discourse a context which consists of a set of what she calls discourse entities. These discourse entities then become available as objects that pronouns can refer to. One of the examples that Webber discusses is the interpretation of the pronoun they in (42) in the context of sentence (41).

(41) Every admiral deployed a ship yesterday.

(42) They arrived.

Clearly they refers to the set of ships deployed by some admiral. What is interesting, of course, about the example is that syntactically there is no plural noun phrase in the preceding discourse that could serve as the referent for the plural pronoun they.

In order to derive the appropriate discourse entity for the interpretation of they. Webber suggests the rule schema as in (43). (43) says that for any formula that meets this structural description, a discourse entity identified by this formula is to be constructed.

(43) SD: $\forall y_{1} \ldots y_{x} \exists \times[P \rightarrow Q]$

ID: $\lambda x \quad \exists y_{1} \ldots y_{k}[P \& Q]$

Instantiated for sentence (41) and its translation (44), the rule produces the expression in (45).

(44) $\forall x \exists y, t, t^{\prime}, t^{n}\left[\right.$ admiral' $(x)(t) \& R_{1}(x)(t) \rightarrow$ $\operatorname{ship}^{\prime}(y)\left(t^{\prime}\right) \& R_{2}(y)\left(t^{\prime}\right) \& t_{r}=\left[D A Y\left(t_{s}\right)-1\right]$ $\& t^{\prime \prime} \varepsilon t_{p} \&$ deploy' $\left.(y)(x)\left(t^{\prime \prime}\right)\right]$

(45) $\lambda$ y $\exists x, t, t^{\prime}, t^{n}\left[\right.$ ship $(y)(t) \& R_{2}(y)(t)$ $\&$ admiral' $(x)\left(t^{\prime}\right) \& R_{1}(x)\left(t^{\prime}\right) \& t_{r}=\left[\operatorname{DAY}\left(t_{s}\right)-1\right]$

$\& t^{n} \varepsilon t_{r} \&$ deploy' $\left.\left.(y)(x)\left(t^{n}\right)\right]\right]$

(45) denotes the set of ships that have been deployed by some admiral. This discourse entity with that description then becomes available for the interpretation of the pronoun they.

It turns out that the method of constructing discourse entities is not only relevant for the interpretation of pronouns, but also for the contextual interpretation of nouns and noun phrases that $I$ am concerned with here.

The discourse entity with the description in (45) cannot only serve for interpreting pronouns, but also for instantiating the contextually specified variable $R$ for the interpretation of the noun ship in (46b) in the context of (46a).

(46) a. Did every admiral deploy a ship yesterday?

b. Which ships will arrive in Hawaii?

Since the discourse entity in (45), which ranges over a set of ships, is described in terms of the property of having been deployed by some admiral the day prior to the day of the speech point, that property can be taken to be salient in the discourse context. If one substitutes the context variable $R$ in the translation (47) of (46b) by this contextually salient property, the temporal evaluation of the predicate ship' in the resulting formula (48) is no longer governed by the existen. tial quantifier $t$ for the future tense, but rather by the quantifier $t^{\prime}$ introduced by the contextually salient property. As a consequence of this instantiation of the context variable $R$, the set of ships under consideration is restricted in the appropriate way. which are assumed to be bound by the discourse context.

(47) QUERY [ $\lambda z$ [ z $\varepsilon$ POW[ $\lambda$ y $\exists t^{\prime}\left[\right.$ ship' $(y)\left(t^{\prime}\right)$

$$
\left.\left.\& R(y)\left(t^{\prime}\right)\right]\right] \& \exists t\left[t>t_{s} \& t \varepsilon t_{r}\right.
$$$$
\left.\& \text { go-to'(Hawaii')(z)(t) }]_{t}\right]
$$

(48) QUERY [ $\lambda z$ [ $z$ \& POW[ $\lambda$ y $\exists t^{\prime}\left[\operatorname{ship}^{\prime}(y)\left(t^{\prime}\right)\right.$

$\& \exists x, t^{\prime \prime}, t^{\prime \prime}\left[\right.$ admiral' $(x)\left(t^{\prime \prime}\right) \& R_{1}(x)\left(t^{\prime \prime}\right) \& t_{t}=$

[DAY $\left.\left(t_{s}\right)-1\right] \& t^{\prime \prime \prime} \varepsilon t_{r} \&$ deploy' $\left.\left.(y)(x)\left(t^{\prime \prime \prime}\right)\right]\right]$

$\left.\& \exists t\left[t>t_{s} \& t \varepsilon t_{p}^{\prime} \& \text { go-to'(Hawaii')(z)(t) }\right]_{t_{s}}\right]$

Notice that (48) contains two reference time parameters $t_{r}$ and $t^{\prime}$, which are associated with quantifiers ranging over past and future times, respectively. I am assuming here that each tense has associated reference time which is updated during discourse processing. $^{6}$

The mechanism for deriving contextually salient properties which are introduced through the previous linguistic discourse may strike the reader as rather complicated in detail. However, as I have argued in this section, tracking such properties is important not only for temporal evaluation, but is independently motivated by other discourse phenomena such as anaphoric reference, as Webber $(1978,1983)$ has convincingly shown.

\section{A Compositional Syntax and Semantics of Tense}

In the previous sections I have focused on the semantic and pragmatic aspects of my analysis of temporal expressions, that concern in particular the feature of narrow scope assignment of tense and the feature of context-dependent interpretation of quantified NP's. In this section I will concentrate on matters of syntax and will demonstrate how the narrow scope analysis of tense makes it possibie to construct a straightforward compositional syntax and semantics of temporal expressions.

Syntactically tenses in English appear as inflec. tional morphemes on verbs. In the notation of categorial grammar, I assign a syntactic tree as in (50) to sentence (49). The untensed form of the verb arrive of category IV is combined with the past tense morpheme -ed to form a tensed intransitive verb IV*. Morpho-syntactically, tenses are therefore items that apply to individual words.

(49) Every ship arrived.

\footnotetext{
'See Hinrichs (1981) for more details on this point.
} 


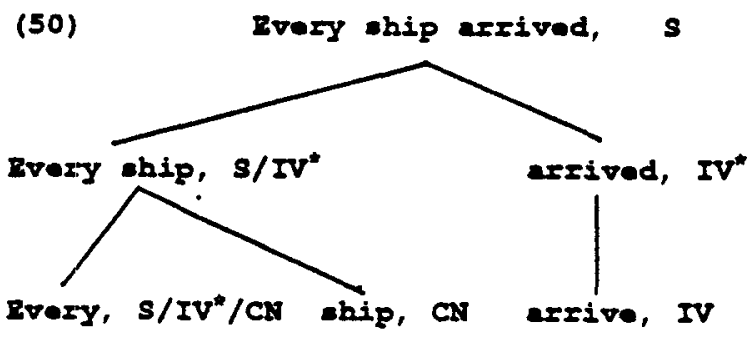

Since $I$ assign tense narrow scope in the semantics and let temporal quantifiers bind only the temporal index associated with the main verb, $I$ arrive at an analysis of tense where its syntactic domain coincides with its semantic domain. Compared to analyses in which tense is assigned wide scope over formulas which correspond to entire sentences (Montague 1973) or over entire verb phrases (Bach 1980), the narrow scope analysis, which I have developed in this paper, has the advantage of leading to a straightiorward compositional syntax and semantics of tense. In the syntax the tense morpheme turns an untensed verb into its tensed counterpart, while in the corresponding translation rule tense has the effect of $6 x$ istentially quantifying over the time-index of the predicate which translates the untensed verb.

(51) S17. If $\alpha \in P_{\text {IVINP }}$ and then $F_{11}(\alpha) \varepsilon P_{I V M N P}$ with $F_{11}=\alpha \cdot \theta d$.

(52) T17. If $\alpha \varepsilon P_{I V M N P}$ and $\alpha$ translates into $\alpha$, then , then $F_{11}(\alpha)$ translates into

$\lambda S_{\uparrow} \ldots S_{n} \lambda \times\left[\exists t^{\prime}\left[t^{\prime}<t_{s} \& t^{\prime} \varepsilon t_{q} \&\right.\right.$ $\left.\alpha^{\prime}\left(S_{1}\right) \ldots\left(S_{n}\right)(x)\left(t^{\prime}\right)\right]$.

$\mathrm{S} 17$ is a rule schema which ranges over untensed intransitive verbs (IV), transitive verbs (IVINP), ditransitive verbs (IV/NP/NP), etc. The notation IV/NNP, thus, stands for an IV followed by $n$ slashed NP's. The corresponding translation schema T17 denotes a function from the type of meanings associated with object NP's, if any, to functions from individuals to truth values. Although these rule schemata are rather technical, their meaning should become clearer, when one considers a concrete example. Consider once again the example (53) whose syntax has been given in $(50)$.

\section{(53) Every ship arrived.}

The translation of the entire sentence can be built up in a compositional fashion as in (54), which mirrors the syntactic composition of (50).

(54) arrived translates as:

$\lambda \times\left[\exists t^{\prime}\left[t^{\prime}<t_{s} \& t^{\prime} \varepsilon t_{p} \&\right.\right.$ arrive' $\left.\left.(x)\left(t^{\prime}\right)\right]\right]$

every translates as:

$\lambda P \lambda Q \forall x[\exists t[P(x)(t) \& R(x)(t)] \rightarrow Q(x)]$

every ship translates as:

$\lambda Q \forall x\left[B t\left[\operatorname{ship}^{\prime}(x)(t) \& R(x)(t)\right] \rightarrow Q(x)\right]$
Every ship arrived translates as:

1. $\lambda Q \forall x\left[\exists t\left[\operatorname{ship}^{\prime}(x)(t) \& R(x)(t)\right] \rightarrow Q(x)\right]$

$\left(\lambda y\left[\exists t^{\prime}\left[t^{\prime}<t_{s} \& t^{\prime} \in t_{p} \&\right.\right.\right.$ arrive' $\left.\left.\left.(y)\left(t^{\prime}\right)\right]\right]\right)$

2. $\forall x\left[\exists t\left[\operatorname{ship}^{\prime}(x)(t) \& R(x)(t)\right] \rightarrow \lambda y\left[\exists t^{\prime}\left[t^{\prime}<t_{s}\right.\right.\right.$ $\left.\& t^{\prime} \varepsilon t, \& \operatorname{arriv} \theta^{\prime}(y)\left(t^{\prime}\right) \prod(x)\right]$

3. $\forall x\left[\exists t\left[\operatorname{ship}^{\prime}(x)(t) \& R(x)(t)\right] \rightarrow \exists t^{\prime}\left[t^{\prime}<t_{s}\right.\right.$ $\left.\& t^{\prime} \varepsilon t, \& \operatorname{arriv}^{\prime}(x)\left(t^{\prime}\right)\right]$

The phrase every ship is formed by supplying the predicate ship' as an argument to the translation of every. Notice that the context-variable $R$ is introduced by the translation of the quantifier every. The translation of the entire sentence is formed by supplying the translation of the tensed verb arrived, which is produced by the translation $\mathrm{T} 17$, to the translation of the subject NP. The reduced translation results from two steps of lambda-reduction.

\section{Conclusion}

In this paper I have argued that a logical semantics for temporal expressions can provide adequate representations for natural language input to an interface such as JANUS. The temporal logic is based on Reichenbach's models for the semantics of English tense and uses multiple indices for semantic interpretation. This multi-indexed logic overcomes the kinds of problems that arise when systems of tense logics are used that rely on just one index of evaluation.

I have demonstrated how giving narrow scope to tense quantifiers enables us to provide adequate scope relations with respect to NP quantifiers and to interpret such NP's relative to a given discourse context. I have argued that the context-dependent feature of the analysis does not add extra complexity to my treatment of time-dependent expressions, but is needed for purposes of discourse understanding in general. Finally, I have demonstrated how the narrow scope of tense results in a fully compositional syntax and semantics of tensed sentences in English.

\section{Acknowledgements}

I am grateful to Remko Scha and Barry Schein for comments on earlier drafts of this paper. My indebtedness to the work of Hans Reichenbach and Murvet Enc on matters of temporal semantics will be evident throughout the paper. 


\section{REFERENCES}

Aqvist, Lennart (1976). 'Formal Semantics for Verb Tenses as Analyzed by Reichenbach'. In: van Dijk, Teun ed. Pragmatics of Language and Literature. Amsterdam: North Holland, pp. 229-236.

Bach, Emmon (1980). "Tenses and Aspect as Functions of Verb Phrases". In Ch. Rohrer ed. Times, Tenses, and Quantifiers. Niemeyer: Tuebingen, W. Germany.

Clark, H. H. and Marshall, C.R. (1981) "Definite Reference and Mutual Knowledge". In: A. Joshi, B. Webber and I. Sag eds. Elements of Discourse Understanding. Cambridge University Press: Cambridge, pp. 10-63.

Dowty, David R. (1982). 'Tenses, Time Adverbs, and Compositional Semantic Theory'. Linguistics and Philosophy Vol.5. pp. 23-55.

Enc, Murvet (1981). Tense without Scope: An Analysis of Nouns as Indexicals. University of Wisconsin, Madison dissertation. Distributed by IULC.

Enc, Murvet (1986). Towards a Referential Analysis of Temporal Expressions". Linguistics and Philosophy. Vol. 9.4. pp. 405-426.

Hinrichs, Erhard (1981). Temporale Anaphora im Englischen. unpublished Staatsexamen thesis: University of Tuebingen.

Hinrichs, Erhard (1986). "Temporal Anaphora in Discourses of English". Linguistics and Philosophy, Vol. 9.1, pp. 63-82.

Hinrichs, Erhard, Damaris Ayuso and Remko Sha (1987). The Syntax and Semantics of a Meaning Representation Language for JANUS". In: Research and Development in Natural Language Understanding as Part of the Strategic Computing Program, Annual Technical Report December 1985 - December 1986, BBN Technical Report 6522.

Link, Godehard (1983). 'The Logical Analysis of Plurals and Mass Terms'. In: Baeuerle. Schwarze and von Stechow eds. Meaning, Use and Interpretation of Language. Berlin: De Gruyter, pp. 250-269.

Montague, Richard (1973). Formal Philosophy. ed. by Richmond Thomason. Yale University Press: New Haven.

Prior, Arthur (1967). Past, Present and Future. Oxford: Oxford University Press.

Partee, Barbara H. (1973). 'Some Structural Analogies between Tenses and Pronouns'. The Journal of Philosophy 70:18, pp. 601-609.

Reichenbach. Hans (1947). Elements of Symbolic Logic. Berkeley: University of California Press.

Scha, Remko (1983). Logical Foundations for Question Answering. Philips Research Laboratories M.S. 12.331. Eindhoven, The Netherlands.
Sidner, Candace (1983). "Focusing the Comprehension of Definite Anaphora". In Brady, Michael and Robert Berwick eds. Computational Models of Discourse. Boston: MIT Press, pp. 267-330.

Stalnaker, Robert (1973). "Pragmatics". In D. Davidson and G. Harman eds. Semantics of Natural Language. Reidel Publishing: Dordrecht, pp. 380-397.

Stalnaker, Robert (1978). "Assertion". In: P. Cole ed. Syntax and Semantics Vol. 9. New York: Academic Press, pp. 315-332.

Webber, Bonnie (1978). A Formal Approach to Discourse Anaphora. BBN Technical Report No. 3761. Bolt Beranek and Newman, Inc.: Cambridge, MA.

Webber, Bonnie (1983). "So what can we talk about now?". Brady, Michael and Robert Berwick eds. Computational Models of Discourse. Boston: MIT Press, pp. $331-\overline{37}$ 